\title{
BAS DU SPECTRE ET DELTA-HYPERBOLICITÉ EN GÉOMÉTRIE DE HILBERT PLANE
}

\author{
par Bruno Colbois \& Constantin Vernicos
}

\begin{abstract}
RÉSUmÉ. - On montre l'équivalence entre l'hyperbolicité au sens de Gromov de la géométrie de Hilbert d'un domaine convexe du plan et la non nullité du bas du spectre de ce domaine.

ABSTRACT (Bottom of the spectrum and delta hyperbolicity in Hilbert plane geometry)

We prove that the Hilbert geometry of a convex domain in the plane is Gromov hyperbolic, if, and only if, the bottom of its spectrum is not zero.
\end{abstract}

\section{Introduction}

Le but de ce travail est de montrer l'équivalence entre l'hyperbolicité au sens de Gromov de la géométrie de Hilbert d'un domaine convexe $\mathcal{C}$ du plan et la non nullité du bas du spectre $\lambda_{1}(\mathcal{C})$ de ce domaine.

Il existe des relations très fortes en géométrie riemannienne entre le bas du spectre du laplacien d'une variété complète de volume infini et la géométrie de cette variété. Par exemple, on sait que le bas du spectre d'une variété de

Texte reçu le 4 novembre 2004, accepté le 14 mars 2005.

Bruno Colbois, Institut de mathématique, Université de Neuchâtel, rue Émile Argand 11, Case Postale 158, 2009 Neuchâtel (Suisse) • E-mail : Bruno.Colbois@unine.ch Constantin Vernicos, Institut de mathématique, Université de Neuchâtel, rue Émile Argand 11, Case Postale 158, 2009 Neuchâtel (Suisse)

E-mail : Constantin.Vernicos@unine.ch

Classification mathématique par sujets (2000). — Géométrie différentielle, géométrie métrique.

Mots clefs. - Géométrie de Hilbert, hyperbolicité, bas du spectre. 
Cartan-Hadamard à courbure sectionnelle $K \leq C<0$ est strictement positif. Récemment, J. Cao [5] a étudié le cas des variétés riemanniennes hyperboliques au sens de Gromov possédant un quasi-pôle, et montré que leur constante de Cheeger (donc le bas de leur spectre) était strictement positif. Dans cet article, nous abordons ce type de questions dans le contexte des géométries de Hilbert.

Avant d'énoncer les résultats précis, rappelons qu'une géométrie de Hilbert $\left(\mathcal{C}, d_{\mathcal{C}}\right)$ est la donnée d'un ouvert convexe et borné $\mathcal{C}$ de $\mathbb{R}^{n}$ muni de la distance de Hilbert $d_{\mathcal{C}}$ définie de la manière suivante : pour toute paire de points distincts $p$ et $q$ dans $\mathcal{C}$, la droite passant par $p$ et $q$ rencontre le bord $\partial \mathcal{C}$ de $\mathcal{C}$ en deux points distincts $a$ et $b$ tels que la droite passe par $a, p, q$ et $b$ dans cet ordre.

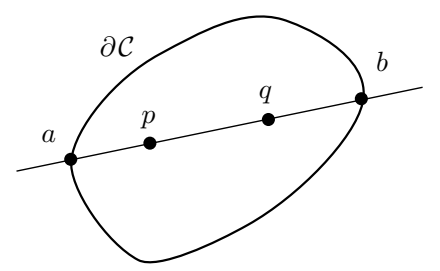

Figure 1. Distance de Hilbert

On définit alors

$$
d_{\mathcal{C}}(p, q)=\frac{1}{2} \ln [a, p, q, b]
$$

où $[a, p, q, b]$ est le birapport de $(a, p, q, b)$ :

$$
[a, p, q, b]=\frac{\|q-a\|_{e}}{\|p-a\|_{e}} \times \frac{\|p-b\|_{e}}{\|q-b\|_{e}}
$$

où $\|.\|_{e}$ désigne la norme euclidienne. On pose également $d_{\mathcal{C}}(p, p)=0$.

Sur $\mathcal{C}$, on peut mettre une norme de Finsler $C^{0}$, notée $\|\cdot\|_{\mathcal{C}}$, en procédant comme suit : si $p \in \mathcal{C}$ et $u_{p} \in T_{p} \mathcal{C}=\mathbb{R}^{n}$, la droite passant par $p$ et dirigée par $u_{p}$ coupe $\partial \mathcal{C}$ en deux points $p^{+}$et $p^{-}$. On pose alors

$$
\left\|u_{p}\right\|_{\mathcal{C}}=\frac{1}{2}\left\|u_{p}\right\|_{e}\left(\frac{1}{\left\|p-p^{+}\right\|_{e}}+\frac{1}{\left\|p-p^{-}\right\|_{e}}\right)
$$

où $\left\|u_{p}\right\|_{e}$ désigne la norme euclidienne de $u_{p}$.

Notons que la distance de longueur induite sur $\mathcal{C}$ par la norme $\|.\|_{\mathcal{C}}$ coïncide avec $d_{\mathcal{C}}$, mais nous n'utiliserons pas ce fait dans la suite.

À cette norme de Finsler est associée une norme duale : si $\ell_{p}$ est une forme linéaire $\operatorname{sur} T_{p} \mathcal{C}$, on pose

$$
\left\|\ell_{p}\right\|_{\mathcal{C}}^{*}=\sup \left\{\ell_{p}\left(u_{p}\right): u_{p} \in T_{p} \mathcal{C},\left\|u_{p}\right\|_{\mathcal{C}}=1\right\} .
$$

TOME $134-2006-\mathrm{N}^{\mathrm{O}} 3$ 
Grâce à la norme de Finsler, on construit une forme volume et une mesure sur $\mathcal{C}$ (qui correspond en fait à la mesure de Hausdorff, voir [4], exemple 5.5.13).

Soient $p \in \mathcal{C}$ et $T B_{\mathcal{C}}(p)=\left\{u_{p} \in T_{p} \mathcal{C}=\mathbb{R}^{n}:\left\|u_{p}\right\|_{\mathcal{C}}<1\right\}$ la boule unité de $T_{p} \mathcal{C}$. Soit $\omega_{n}$ le volume de la boule unité de l'espace euclidien $\mathbb{R}^{n}$ et considérons la fonction $h: \mathcal{C} \rightarrow \mathbb{R}$ donnée par $h(p)=\omega_{n} / \operatorname{vol}_{e}\left(T B_{\mathcal{C}}(p)\right)$ où $\operatorname{vol}_{e}$ est le volume euclidien usuel.

Alors la mesure $\mu_{\mathcal{C}}$ (que nous nommerons mesure de Hilbert de $\mathcal{C}$; elle est également connue sous le nom de mesure de Busemann) associée à $\|.\|_{\mathcal{C}}$ est définie ainsi : si $A \subset \mathcal{C}$ est un borélien, on pose

$$
\mu_{\mathcal{C}}(A)=\int_{A} h(p) \operatorname{dvol}_{e}(p)
$$

où $\operatorname{dvol}_{e}(p)$ est la mesure de Lebesgue.

Enfin, si $\Omega$ est un domaine avec $\bar{\Omega} \subset \mathcal{C}$, l'intégrale sur $\Omega$ par rapport à $\mu_{\mathcal{C}}$ d'une fonction $f$ définie sur $\Omega$ sera notée $\int_{\Omega} f \mathrm{~d} \mu_{\mathcal{C}}$.

REMARQUE. - Les résultats de cet article restent inchangés si on considère une mesure équivalente. En particulier, la mesure dite de Holmes-Thompson, qui est équivalente par les inégalités de Santalo et Bourgain-Milman à la mesure de Hilbert, donne les mêmes résultats.

Lorsque $\mathcal{C}$ est une ellipse, $\left(\mathcal{C}, d_{\mathcal{C}}\right)$ est le modèle projectif (ou modèle de Klein) de la géométrie hyperbolique, et on peut penser aux géométries de Hilbert $\left(\mathcal{C}, d_{\mathcal{C}}\right)$ comme à une généralisation naturelle de l'espace hyperbolique. Une question communes à de nombreux travaux récents (voir [13], [14], [1], [8], [7], [11] et leurs références) est de déterminer les propriétés de l'espace hyperbolique dont ces géométries héritent et de trouver des caractérisations de l'espace hyperbolique parmi les géométries de Hilbert. En particulier, Y. Benoist [1] obtient en toute dimension une caractérisation des convexes dont la géométrie de Hilbert associée est hyperbolique au sens de Gromov (voir [3] pour une discussion de ce concept). Cette caractérisation est donnée en fonction de la régularité du bord des convexes considérés.

Dans cet article, nous allons caractériser en dimension 2 l'hyperbolicité au sens de Gromov d'un point de vue spectral : nous montrons qu'en dimension 2 avoir un bas du spectre strictement positif est équivalent à être hyperbolique au sens de Gromov.

On définit le bas du spectre de $\mathcal{C}$, que l'on note $\lambda_{1}(\mathcal{C})$, par analogie avec ce qui se fait dans le cas des variétés riemanniennes de volume infini. On pose

$$
\lambda_{1}(\mathcal{C})=\inf \frac{\int_{\mathcal{C}}\left\|\mathrm{d} f_{p}\right\|_{\mathcal{C}}^{* 2} \mathrm{~d} \mu_{\mathcal{C}}(p)}{\int_{\mathcal{C}} f^{2}(p) \mathrm{d} \mu_{\mathcal{C}}(p)}
$$

BULletin DE LA SOCiÉtÉ MATHÉmATiQUE DE FRANCE 
où l'infimum est pris sur toutes les fonctions lipschitziennes, non nulles, à support compact dans $\mathcal{C}$ et où $\mu_{\mathcal{C}}$ est la mesure de Hilbert associée à $\mathcal{C}$. L'expression ci-dessus est appelée le quotient de Rayleigh de $f$.

Le bas du spectre $\lambda_{1}(\mathcal{C})$ est un nombre réel positif ou nul. On sait que lorsque $\mathcal{C}$ est une ellipse, c'est-à-dire que l'on se trouve dans le modèle hyperbolique, il vaut $\frac{1}{4}$. On verra un peu plus loin que dans le cas où $\mathcal{C}$ est un triangle, le bas du spectre est nul.

Notre résultat principal est :

ThÉORÈme 0.1. - Soit $\left(\mathcal{C}, d_{\mathcal{C}}\right)$ un convexe du plan muni de sa métrique de Hilbert. Alors son bas du spectre est non nul, i.e., $\lambda_{1}(\mathcal{C}) \neq 0$, si et seulement s'il existe $\delta>0$ tel que $\left(\mathcal{C}, d_{\mathcal{C}}\right)$ est $\delta$-hyperbolique.

Pour un domaine ouvert, borné (relativement à $d_{\mathcal{C}}$ ) $\Omega$ à bord lipschitzien de $\mathcal{C}$, on appellera bas du spectre de Dirichlet le nombre

$$
\lambda_{1}^{\mathcal{C}}(\Omega)=\inf \frac{\int_{\Omega}\left\|\mathrm{d} f_{p}\right\|_{\mathcal{C}}^{* 2} \mathrm{~d} \mu_{\mathcal{C}}(p)}{\int_{\Omega} f^{2}(p) \mathrm{d} \mu_{\mathcal{C}}(p)},
$$

où l'infimum est pris sur toutes les fonctions lipschitziennes, non nulles, à support dans $\Omega$.

Il est clair que $\lambda_{1}^{\mathcal{C}}(\Omega) \geq \lambda_{1}(\mathcal{C})$ et que $\lambda_{1}^{\mathcal{C}}$ est une fonction décroissante suivant l'inclusion des ensembles : en effet, si $\Omega_{1} \subset \Omega_{2}$, toute fonction à support dans $\Omega_{1}$ s'étend naturellement par 0 en une fonction à support dans $\Omega_{2}$.

L'idée de la preuve du théorème 0.1 est la suivante.

À la section 1, on montrera que la non nullité du bas du spectre entraîne l'hyperbolicité au sens de Gromov en nous appuyant principalement sur un résultat de Y. Benoist qui donne une condition suffisante pour qu'une famille de convexes munis de leur métrique de Hilbert soit $\delta$-hyperbolique (proposition 1.2 ci-dessous). On montrera que tous les convexes dont le bas du spectre est supérieur ou égal à une constante positive $\lambda$ donnée sont $\delta$-hyperboliques, $\delta$ dépendant de $\lambda$.

À la section 2 , on montrera la réciproque en utilisant une inégalité isopérimétrique que vérifie les espaces $\delta$-hyperboliques et sur une estimation du volume des boules, intéressante pour elle-même, valable en toute dimension, donnée à la section 2.1. En particulier, le volume d'une boule de rayon fixé est uniformément majoré sur toutes les géométries de Hilbert de dimension $n$. Dans le cas riemannien, cela est impliqué par la donnée d'un minorant sur la courbure de Ricci, mais on sait qu'une hypothèse de ce type n'est pas vérifiée par les géométries de Hilbert qui ne sont pas, en général, des espaces d'Alexandroff. Pour conclure, nous aurons besoin d'une inégalité du type Cheeger reliant les

TOME $134-2006-\mathrm{N}^{\mathrm{O}} 3$ 
constantes isopérimétriques et le bas du spectre. Pour l'essentiel, on peut adapter ce qui se fait dans la cas riemannien en faisant un détour par le cas finslérien, mais cela pose quelques problèmes techniques. D'une part, les convexes que l'on considère possèdent une métrique de Finsler seulement $C^{0}$, ce qui ne permet pas sans autre d'adapter l'inégalité de Cheeger riemannienne, d'autre part, il y a différentes façons d'induire une mesure sur les hypersurfaces selon que l'on s'intéresse à l'inégalité isopérimétrique ou à la formule de la co-aire (comme souvent ces notions coïncident dès que l'on se trouve dans un contexte riemannien). Aussi avons nous traité ce problème d'un point du vue général des variétés de Finsler aux sections 3 (pour la formule de la co-aire) et 4 (pour l'inégalité de Cheeger). Ces deux sections sont intéressantes pour elles-mêmes, mais peuvent également être admises pour la preuve du théorème 0.1

\section{La non nullité du bas du spectre implique la $\delta$-hyperbolicité}

ThÉORÈme 1.1. - Un convexe $\left(\mathcal{C}, d_{\mathcal{C}}\right)$ du plan, muni de sa métrique de Hilbert et dont le bas du spectre est non nul est Gromov hyperbolique. Plus précisément :

$$
\forall \lambda>0, \exists \delta>0 \text { tel que } \forall \mathcal{C}, \lambda_{1}(\mathcal{C}) \geq \lambda \Longrightarrow\left(\mathcal{C}, d_{\mathcal{C}}\right) \text { est } \delta \text {-hyperbolique. }
$$

Soient $G_{n}:=\operatorname{PGL}\left(\mathbb{R}^{n+1}\right)$ et $\mathbb{P}^{n}:=\mathbb{P}\left(\mathbb{R}^{n+1}\right)$ l'espace projectif de $\mathbb{R}^{n+1}$.

Une partie proprement convexe $\mathcal{C}$ de $\mathbb{P}^{n}$ est une partie convexe dont l'adhérence est incluse dans le complémentaire d'un hyperplan projectif. Ainsi on peut lui associer un convexe borné de $\mathbb{R}^{n}$ et, inversement, à un convexe borné de $\mathbb{R}^{n}$ on peut associer une partie proprement convexe de $\mathbb{P}^{n}$. Dans la suite, on désignera par $X_{n}$ l'ensemble des ouverts proprement convexes et par $X_{n}^{\delta}$ l'ensemble des ouverts proprement convexes et $\delta$-hyperboliques de $\mathbb{P}^{n}$, munis de leur métrique de Hilbert.

Nous munissons $X_{n}$ de la distance de Hausdorff entre les ensembles, comme définie dans [1], p. 2.

On peut alors énoncer :

Proposition 1.2 (cf. [1, prop. 2.11]). - Soit $F$ un sous ensemble fermé de $X_{n}, G_{n}$-invariant, dont tous les éléments sont strictement convexes (c'est$\grave{a}$-dire que l'intérieur du segment reliant deux points du bord de $\mathcal{C}$ est dans $\mathcal{C}$ ). Alors il existe un nombre réel $\delta>0$ tel que $F \subset X_{n}^{\delta}$.

En sorte que le théorème 1.1 se déduit aisément de la proposition 1.2 grâce à la proposition suivante.

Proposition 1.3. - Soit $\lambda>0$ et $F_{\lambda} \subset X_{n}$ l'ensemble des convexes de $X_{n}$ dont le bas du spectre est supérieur ou égal à $\lambda$. Alors $F_{\lambda}$ est une partie fermée et $G_{n}$-invariante de $X_{n}$, et si $n=2$, tous ses éléments sont strictement convexes. 
L'idée de la preuve de cette proposition est la suivante :

- La $G_{n}$-invariance est immédiate, car pour tout $g \in G_{n}$ et $\mathcal{C} \in X_{n}, \mathcal{C}$ et $g \mathcal{C}$ sont des espaces métriques isométriques, et ils ont donc le même bas du spectre.

- La difficulté principale résidera dans la fermeture. Supposons qu'il existe une suite $\left(\mathcal{C}_{i}\right)$ dans $F_{\lambda}$ telle que $\mathcal{C}_{i}$ converge vers $\mathcal{C}$ au sens de Hausdorff dans $X_{n}$ et $\lambda_{1}(\mathcal{C})<\lambda$. Alors, pour tout $\varepsilon>0$, on va montrer qu'il existe un domaine $\Omega$ tel que $\bar{\Omega} \subset \mathcal{C}$ avec $\lambda_{1}^{\mathcal{C}}(\Omega) \leq \lambda_{1}(\mathcal{C})+\varepsilon$. Par ailleurs, pour $i$ assez grand, on aura $\bar{\Omega} \subset \mathcal{C}_{i}$. On en déduira que $\lambda_{1}\left(\mathcal{C}_{i}\right)<\lambda$, ce qui est une contradiction.

- Le fait que la limite $\mathcal{C}$ est strictement convexe découlera alors directement.

On formalise cette idée intuitive à l'aide des deux lemmes suivants.

Lemme 1.4. - Soit $\mathcal{C} \subset \mathbb{R}^{n}$ un domaine convexe. Alors, pour tout $\varepsilon>0$, il existe un domaine $\Omega$ non vide avec $\bar{\Omega} \subset \mathcal{C}$ tel que

$$
\lambda_{1}^{\mathcal{C}}(\Omega) \leq \lambda_{1}(\mathcal{C})+\varepsilon .
$$

Démonstration. - Fixons un point origine $P_{0} \in \mathcal{C}$. Par définition de $\lambda_{1}(\mathcal{C})$, il existe une fonction $f$ à support dans $\mathcal{C}$ de quotient de Rayleigh inférieur à $\lambda_{1}(\mathcal{C})+\varepsilon$. La distance euclidienne entre le support de $f$ et le bord $\partial \mathcal{C}$ de $\mathcal{C}$ étant strictement positif, il existe $\alpha<1$ tel que $f$ est encore à support dans un homothétique $\alpha \mathcal{C}$ de $\mathcal{C}$ par rapport au point $P_{0}$.

On choisit alors $\Omega=\alpha \mathcal{C}$, et, toujours par définition, le bas du spectre de $\Omega$ (comme domaine de $\mathcal{C}$ ) est majoré par le quotient de Rayleigh de $f$, donc $\operatorname{par} \lambda_{1}(\mathcal{C})+\varepsilon$.

Notation. - Soit $\mathcal{C}$ un domaine convexe et $P_{0} \in \mathcal{C}$. Pour $\rho>0$, on va considérer les convexes $(1+\rho) \mathcal{C}$ et $(1-\rho) \mathcal{C}$ qui sont les homothétiques de $\mathcal{C}$ par une homothétie de centre $P_{0}$ et de rapport $1+\rho$ et $1-\rho$ respectivement.

Lemme 1.5. - Soit $\mathcal{C}$ un domaine convexe et $\Omega$ un domaine tel que $\bar{\Omega} \subset \mathcal{C}$. Soit $\varepsilon>0$. Alors, il existe $0<\rho<1$ tel que $\bar{\Omega} \subset(1-\rho) \mathcal{C}$ et tel que pour tout convexe $\Gamma$ vérifiant $(1-\rho) \mathcal{C} \subset \Gamma \subset(1+\rho) \mathcal{C}$, on ait

$$
(1-\varepsilon) \lambda_{1}^{\mathcal{C}}(\Omega) \leq \lambda_{1}^{\Gamma}(\Omega) \leq(1+\varepsilon) \lambda_{1}^{\mathcal{C}}(\Omega) .
$$

Démonstration. - Comme au lemme précédent, on montre qu'il existe $\rho_{0}>0$ avec

$$
\bar{\Omega} \subset\left(1-2 \rho_{0}\right) \mathcal{C} \subset\left(1-\rho_{0}\right) \mathcal{C} .
$$

Ainsi, pour tout $0<\rho<\rho_{0}$, la distance euclidienne entre $\bar{\Omega}$ et $\partial((1-\rho) \mathcal{C})$ est uniformément minorée par une constante strictement positive.

Dès maintenant, on suppose $\rho<\rho_{0}$ et $(1-\rho) \mathcal{C} \subset \Gamma \subset(1+\rho) \mathcal{C}$.

Soit $p \in \Omega$ et $u_{p} \in T_{p} \mathcal{C}$, un vecteur non nul. Comme conséquence directe de la définition de la norme de Finsler associée à la distance de Hilbert, on a

$$
\left\|u_{p}\right\|_{(1+\rho) \mathcal{C}} \leq\left\|u_{p}\right\|_{\Gamma} \leq\left\|u_{p}\right\|_{(1-\rho) \mathcal{C}} .
$$

TOME $134-2006-\mathrm{N}^{\mathrm{O}} 3$ 
On en déduit d'une part l'inégalité suivante, par dualité, pour une forme linéaire non nulle $\ell_{p}$ sur $T_{p} \mathcal{C}$,

$$
\left\|\ell_{p}\right\|_{(1-\rho) \mathcal{C}}^{*} \leq\left\|\ell_{p}\right\|_{\Gamma}^{*} \leq\left\|\ell_{p}\right\|_{(1+\rho) \mathcal{C}}^{*},
$$

d'autre part la relation d'inclusion vérifiée par les boules unités de l'espace tangent en $p$ :

$$
T B_{(1-\rho) \mathcal{C}}(p) \subset T B_{\Gamma}(p) \subset T B_{(1+\rho) \mathcal{C}}(p) .
$$

Ce qui implique que les densités de volume associées vérifient

$$
\frac{\omega_{n}}{\operatorname{vol}_{e}\left(B_{(1+\rho) C}(p)\right)} \leq \frac{\omega_{n}}{\operatorname{vol}_{e}\left(B_{\Gamma}(p)\right)} \leq \frac{\omega_{n}}{\operatorname{vol}_{e}\left(B_{(1-\rho) \mathcal{C}}(p)\right)} .
$$

De plus, par compacité de $\Omega$ et par continuité, les rapports

$$
\frac{\left\|u_{p}\right\|_{(1+\rho) \mathcal{C}}}{\left\|u_{p}\right\|_{(1-\rho) \mathcal{C}}}, \frac{\left\|\ell_{p}\right\|_{(1-\rho) \mathcal{C}}^{*}}{\left\|\ell_{p}\right\|_{(1+\rho) \mathcal{C}}^{*}} \text { et } \frac{\operatorname{vol}_{e}\left(B_{(1+\rho) C}(p)\right)}{\operatorname{vol}_{e}\left(B_{(1-\rho) \mathcal{C}}(p)\right)}
$$

sont uniformément contrôlés en fonction de $\rho$ par des fonctions tendant vers 1 lorsque $\rho \rightarrow 0$. Cela étant indépendant du choix de $\Gamma$, on peut comparer deux convexes quelconques du type de $\Gamma$, soit ici $\Gamma$ et $\mathcal{C}$, et on obtient l'existence d'une fonction $h=h(\rho)$ telle que $h(\rho) \rightarrow 0$ lorsque $\rho \rightarrow 0$ et telle que pour une fonction $f$ de classe $C^{\infty}$ à support dans $\Omega$, on ait

$$
(1-h(\rho)) \lambda_{1}^{\mathcal{C}}(\Omega) \leq \lambda_{1}^{\Gamma}(\Omega) \leq(1+h(\rho)) \lambda_{1}^{\mathcal{C}}(\Omega) .
$$

Il suffit donc de choisir $\rho$ assez proche de 0 pour conclure.

Démonstration de la proposition 1.3. - Pour voir que $F_{\lambda}$ est fermé, on considère une suite $\left(\mathcal{C}_{i}\right)_{i \in \mathbb{N}}$ dans $F_{\lambda}$ qui converge vers un convexe $\mathcal{C}$ au sens de Hausdorff. Supposons que $\mathcal{C} \notin F_{\lambda}$, c'est-à-dire qu'il existe $\varepsilon>0$ avec $\lambda_{1}(\mathcal{C}) \leq$ $\lambda-\varepsilon$. Alors, par le premier lemme, il existe un domaine $\Omega$ avec $\bar{\Omega} \subset \mathcal{C}$ et

$$
\lambda_{1}^{\mathcal{C}}(\Omega) \leq(\lambda-\varepsilon)+\frac{1}{3} \varepsilon=\lambda-\frac{2}{3} \varepsilon .
$$

Le deuxième lemme implique l'existence de $\rho>0$ tel que pour tout convexe $\Gamma$ avec $(1-\rho) \mathcal{C} \subset \Gamma \subset(1+\rho) \mathcal{C}$, on ait

$$
\lambda_{1}^{\Gamma}(\Omega) \leq\left(1+\frac{1}{2} \varepsilon\right) \lambda_{1}^{\mathcal{C}}(\Omega) \leq\left(1-\frac{1}{6} \varepsilon\right) \lambda<\lambda .
$$

Cela est en particulier vrai pour les éléments $\mathcal{C}_{i}$ de la suite lorsque $i$ est assez grand et contredit le fait que $\mathcal{C}_{i} \in F_{\lambda}$.

Il reste alors à montrer que tous les convexes de $F_{\lambda}$ sont strictement convexes en dimension deux. Cependant si un convexe n'est pas strictement convexe, il admet un triangle dans l'adhérence de son orbite (voir [1], corollaire 2.9, dû à Benzécri [2]), et on obtient une contradiction comme dans la première partie, car le bas du spectre d'un triangle est nul. On montre ce dernier point par un calcul direct, à cause de la simplicité de la géométrie de Hilbert du triangle. Si on considère une boule $B_{R}$ de rayon $R$, on voit que son aire est de l'ordre de $R^{2}$ et la longueur de son bord de l'ordre de $R$ lorsque $R \rightarrow \infty$. On construit 
alors une fonction test valant 1 sur $B_{R}, 0$ hors de $B_{R+1}$, et valant $1-d\left(p, B_{R}\right)$ pour $p \in B_{R+1}-B_{R}$. Le quotient de Rayleigh d'une telle fonction est de l'ordre de $1 / R$ lorsque $R \rightarrow \infty$.

REMARQUE 1.6. - En ce qui concerne les triangles, une autre manière de voir que le bas du spectre est nul, est d'utiliser le théorème 5 dans [16], qui généralise l'inégalité de Faber-Krahn aux espaces vectoriels normés de dimension finie. Celui-ci permet de dire que le $\lambda_{1}$ d'une boule de rayon $R$ est de l'ordre de $1 / R^{2}$, puisque la géométrie du triangle est isométrique à celle du plan muni de la norme dont la boule unité est un hexagone régulier (voir [9]).

\section{De la $\delta$-hyperbolicité à la non nullité du bas du spectre}

Le but de cette section est la démonstration du théorème suivant :

ThÉORÈme 2.1. - Un convexe du plan $\mathcal{C}$ qui, muni de sa métrique de Hilbert $d_{\mathcal{C}}$ est Gromov-hyperbolique, a son bas du spectre strictement positif. Plus précisément : pour tout $\delta>0$, il existe $\lambda>0$ tel que, pour tout convexe du plan $C$,

$$
\left(\mathcal{C}, d_{\mathcal{C}}\right) \text { est } \delta \text {-hyperbolique } \Longrightarrow \lambda_{1}(\mathcal{C}) \geq \lambda \text {. }
$$

Un point essentiel de la preuve est de contrôler le volume des boules de $\left(\mathcal{C}, d_{\mathcal{C}}\right)$, et nous traitons ce point pour lui-même.

2.1. Volume des boules. - On se propose ici de montrer qu'en géométrie de Hilbert, le volume des boules est contrôlé par leur rayon, et cela indépendemment de la géométrie du convexe considéré.

Notation. - Dans la suite, puisqu'il n'y aura plus d'ambiguïté sur le convexe que l'on considère, on indicera plus les boules par le convexe $\mathcal{C}$ dans lequel elles se trouvent, mais par leur rayon.

ThÉORÈme 2.2. - Il existe deux constantes positives $C_{1}(R, n), C_{2}(R, n)$ telles que si $\mathcal{C}$ est un ouvert convexe borné de $\mathbb{R}^{n}$ muni de sa distance de Hilbert usuelle $d_{\mathcal{C}}$ et de sa mesure finslérienne associée $\mu_{\mathcal{C}}$, alors, pour toute boule $B_{R}$ de rayon $R$ dans $\left(\mathcal{C}, d_{\mathcal{C}}\right)$, on a

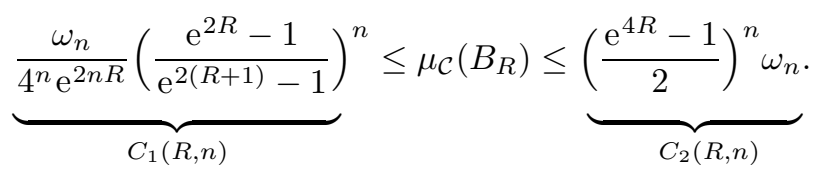

On constate en particulier que lorsque $R \rightarrow 0$, le volume d'une boule de rayon $R$ dans $\mathcal{C}$ est de l'ordre de $R^{n}$, à des constantes multiplicatives près ne dépendant que de la dimension. En particulier, le volume d'une boule de rayon fixé est contrôlé indépendamment de la position de son centre et de $\mathcal{C}$.

TOME $134-2006-\mathrm{N}^{\mathrm{O}} 3$ 
La première étape de la preuve consiste à comparer le volume euclidien d'une boule de rayon $R$ dans $\mathcal{C}$ centrée en $x$ et que l'on note $B_{R}(x)$ et le volume euclidien de la boule de rayon $R$ tangente au point $x$ (pour la métrique de Finsler $F_{\mathcal{C}}$ de classe $C^{0}$ ) qui sera notée $T B_{R}(x)$.

Proposition 2.3. - Il existe deux constantes positives $c_{1}(R, n), c_{2}(R, n)$ (indépendantes de $\mathcal{C}$ et de $x$ ) telles que

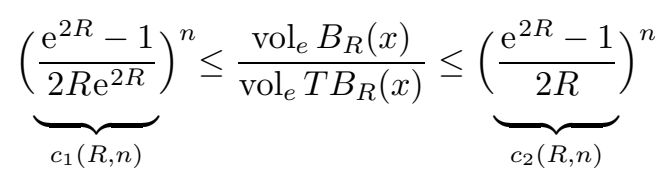

où $\mathrm{vol}_{e}$ désigne le volume euclidien.

Démonstration de la proposition 2.3. - On regarde se qui se passe dans une direction donnée, le long d'une droite. Pour alléger les notations et simplifier les calculs, on va travailler en dimension 1 et supprimer autant que possible les normes intervenant dans l'expression de la métrique de Finsler.

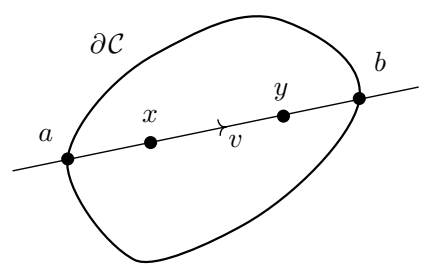

Figure 2. Comparaison de $\|v\|_{e}$ et $y-x$

On se trouve sur une droite avec trois points ordonnés $a, x, b$. Un point $y>x$ tel que $d_{\mathcal{C}}(x, y)=R$ est tel que

$$
\frac{1}{2} \ln \left(\frac{y-a}{x-a} \frac{b-x}{b-y}\right)=R
$$

et un vecteur $v \in T_{x} \mathcal{C}$ pointant vers $b$ et de norme de Finsler $\|v\|_{\mathcal{C}}=R$ sera tel que

$$
\frac{1}{2}\|v\|_{e}\left(\frac{1}{b-x}+\frac{1}{x-a}\right)=R
$$

On cherche à comparer $\|v\|_{e}$ et $y-x$.

Commençons par étudier $y-x$. Puisque $d_{\mathcal{C}}(x, y)=R$, en prenant l'exponentielle de l'équation (6) on obtient

$$
\frac{y-a}{x-a} \cdot \frac{b-x}{b-y}=\mathrm{e}^{2 R}
$$

BULLETIN DE LA SOCIÉtÉ MATHÉMATIQUE DE FRANCE 
puis en isolant $y$,

$$
y=\frac{a(b-x)+\mathrm{e}^{2 R} b(x-a)}{(b-x)+\mathrm{e}^{2 R}(x-a)}
$$

on en déduit alors une expression de $y-x$ en fonction de $a, b, x$ et $R$ :

$$
y-x=\frac{(x-a)(b-x)\left(e^{2 R}-1\right)}{(b-x)+e^{2 R}(x-a)} .
$$

On exprime finalement $y-x$ en fonction des nouvelles variables $\alpha=x-a$, $\beta=b-x$ et $\gamma=b-a$ :

$$
y-x=\frac{\alpha \beta\left(\mathrm{e}^{2 R}-1\right)}{\beta+\mathrm{e}^{2 R} \alpha}=\frac{\alpha \beta\left(\mathrm{e}^{2 R}-1\right)}{\gamma+\left(\mathrm{e}^{2 R}-1\right) \alpha} .
$$

En ce qui concerne $\|v\|_{e}$, en combinant l'expression de la norme de Finsler (7) et le fait que $\|v\|_{\mathcal{C}}=R$, on obtient en fonction de $\alpha, \beta$, $\gamma$ et $R$ :

$$
\|v\|_{e}=\frac{2 R(x-a)(b-x)}{b-a}=\frac{2 R \alpha \beta}{\gamma} .
$$

On utilise à présent les expressions (9) de $\|v\|_{e}$ et (8) de $y-x$ pour obtenir une équation de leur rapport en fonction de $\alpha, \beta$, $\gamma$ et $R$ :

$$
\frac{\|v\|_{e}}{y-x}=\frac{2 R \alpha \beta}{\gamma} \frac{\gamma+\left(\mathrm{e}^{2 R}-1\right) \alpha}{\alpha \beta\left(\mathrm{e}^{2 R}-1\right)}=\frac{2 R}{\left(e^{2 R}-1\right)} \frac{\gamma+\left(\mathrm{e}^{2 R}-1\right) \alpha}{\gamma} .
$$

On remarque alors qu'on a l'inégalité

$$
1 \leq \frac{\gamma+\left(\mathrm{e}^{2 R}-1\right) \alpha}{\gamma} \leq \mathrm{e}^{2 R}
$$

et on l'applique à l'équation (10) pour trouver

$$
\frac{2 R}{\left(\mathrm{e}^{2 R}-1\right)} \leq \frac{\|v\|_{e}}{y-x} \leq \frac{2 R \mathrm{e}^{2 R}}{\left(e^{2 R}-1\right)} .
$$

Cette dernière inégalité induit les inclusions

$$
\frac{2 R}{\left(\mathrm{e}^{2 R}-1\right)} B_{R}(x) \subset T B_{R}(x) \subset \frac{2 R \mathrm{e}^{2 R}}{\left(\mathrm{e}^{2 R}-1\right)} B_{R}(x) .
$$

Les volumes euclidiens respectifs de $B_{R}(x)$ et $T B_{R}(x)$ sont donc contrôlés par les constantes annoncées dans l'énoncé de la proposition, et qui ne dépendent que de $R$ et de $n$.

Corollaire 2.4. - Comme la boule $T B_{R}(x)$ est homothétique de rapport $R$ avec la boule $T B_{1}(x)$, on déduit l'existence de deux constantes positives $c_{1}^{\prime}(R, n)$ et $c_{2}^{\prime}(R, n)$ telles que

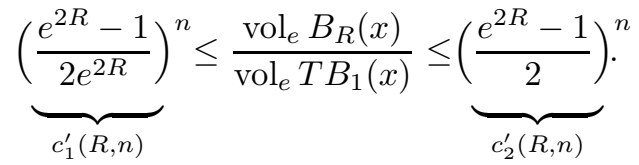

TOME $134-2006-\mathrm{N}^{\mathrm{O}} 3$ 
Démonstration du théorème 2.2. - On cherche à contrôler le volume de la boule $B_{R}(x)$. On sait qu'il est défini par

$$
\mu_{\mathcal{C}}\left(B_{R}(x)\right)=\int_{B_{R}(x)} \frac{w_{n}}{\operatorname{vol}_{e} T B_{1}(y)} \mathrm{d} y
$$

où $\mathrm{d} y$ désigne la mesure de Lebesgue et $\omega_{n}$ le volume euclidien de la boule unité.

On sait par ailleurs que pour tout $y \in B_{R}(x)$, on a $B_{R}(x) \subset B_{2 R}(y)$. En combinant ceci avec le corollaire 2.4, on obtient d'abord

$$
\frac{1}{\operatorname{vol}_{e} T B_{1}(y)} \leq c_{2}^{\prime}(2 R, n) \frac{1}{\operatorname{vol}_{e} B_{2 R}(y)} \leq c_{2}^{\prime}(2 R, n) \frac{1}{\operatorname{vol}_{e} B_{R}(x)},
$$

et puis, par intégration,

$$
\mu_{\mathcal{C}}\left(B_{R}(x)\right) \leq \omega_{n} c_{2}^{\prime}(n, R)=C_{2}(R, n) .
$$

On a également $\operatorname{vol}_{e} T B_{R+1}(x)=(R+1)^{n} \operatorname{vol}_{e} T B_{1}(x)$. Comme $B_{1}(y)$ est inclus dans $B_{R+1}(x)$, suivant le corollaire 2.4 , on a d'une part

$$
\frac{1}{\operatorname{vol}_{e} T B_{1}(y)} \geq\left(\frac{\mathrm{e}^{2}-1}{2 \mathrm{e}^{2}}\right)^{n} \frac{1}{\operatorname{vol}_{e} B_{1}(y)} \geq \frac{1}{4^{n}} \frac{1}{\operatorname{vol}_{e} B_{R+1}(x)},
$$

et d'autre part

$$
\begin{aligned}
\frac{1}{\operatorname{vol}_{e} B_{R+1}(x)} \geq & \left(\frac{2}{\mathrm{e}^{2(R+1)}-1}\right)^{n} \frac{1}{\operatorname{vol}_{e} T B_{1}(x)} \\
& \geq\left(\frac{2}{\mathrm{e}^{2(R+1)}-1}\right)^{n}\left(\frac{\mathrm{e}^{2 R}-1}{2 \mathrm{e}^{2 R}}\right)^{n} \frac{1}{\operatorname{vol}_{e} B_{R}(x)} .
\end{aligned}
$$

On obtient alors par intégration :

$$
\mu_{\mathcal{C}}\left(B_{R}(x)\right) \geq \frac{\omega_{n}}{4^{n} \mathrm{e}^{2 n R}}\left(\frac{\mathrm{e}^{2 R}-1}{\mathrm{e}^{2(R+1)}-1}\right)^{n}=C_{1}(R, n) .
$$

2.2. Constante de Cheeger et bas du spectre. - Dans la suite, on va étudier la constante de Cheeger des sections planaires d'un convexe $\mathcal{C}$ muni de sa métrique de Hilbert. Si $\mathcal{C}$ est un domaine convexe de $\mathbb{R}^{2}$, la constante de Cheeger de $\left(\mathcal{C}, d_{\mathcal{C}}\right)$ est définie par

$$
I_{\infty}(\mathcal{C})=\inf _{\Omega \subset \mathcal{C}} \frac{\nu_{\mathcal{C}}(\partial \Omega)}{\mu_{\mathcal{C}}(\Omega)}
$$

où $\Omega$ est un domaine dont l'adhérence (prise dans $\mathbb{R}^{2}$ ) vérifie $\bar{\Omega} \subset \mathcal{C}$ et dont le bord $\partial \Omega$ est une courbe rectifiable de longueur $\nu_{\mathcal{C}}(\partial \Omega)$.

BULLETIN DE LA SOCIÉtÉ MATHÉMATIQUE DE FRANCE 
On va d'abord montrer que la constante de Cheeger d'un convexe $\left(\mathcal{C}, d_{\mathcal{C}}\right)$ hyperbolique au sens de Gromov est strictement positive.

Proposition 2.5. - Soit $\left(\mathcal{C}, d_{h}\right)$ un convexe du plan muni de sa métrique de Hilbert qui est $\delta$-hyperbolique. Alors il existe une constante $I(\delta)>0$, dépendant de $\delta$, telle que $I_{\infty}(\mathcal{C}) \geq I(\delta)$.

Pour montrer cela on utilise un résultat décrit par M. Bridson et A. Haefliger (cf. Chapitre III.H, section 2, définition 2.1 et proposition 2.7 de [3]) :

Proposition 2.6 (voir [3]). - Soit X un espace géodésique. Si X est $\delta$-hyperbolique, alors il existe deux constantes $A$ et $B$ et $\varepsilon>0$ telles que pour toute courbe rectifiable $c: S^{1} \rightarrow X$ on ait

$$
\operatorname{Aire}_{\varepsilon}(c) \leq A \nu_{X}(c)+B
$$

où $\operatorname{Aire}_{\varepsilon}(c)=\min \{|\Phi| ; \Phi$ est un $\varepsilon$-remplissage de $c\}$ et $\nu_{X}(c)$ est la longueur de la courbe $c$.

Démonstration de la proposition 2.5. - Pour un domaine $\Omega$ de $\mathcal{C}$ de bord rectifiable, on cherche à minorer le quotient $\nu_{\mathcal{C}}(\partial \Omega) / \mu_{\mathcal{C}}(\Omega)$. On peut d'une part supposer que $\Omega$ est connexe, mais également qu'il est simplement connexe. En effet, comme $\Omega$ est borné dans $\mathcal{C}$, son complémentaire dans $\mathcal{C}$ compte exactement une composante connexe non bornée, du fait que $\mathcal{C}$ est homéomorphe à $\mathbb{R}^{2}$. En considérant le complémentaire de cette composante connexe, on obtient un nouveau domaine borné et simplement connexe $\Omega^{\prime}$ contenant $\Omega$, donc vérifiant $\mu_{\mathcal{C}}\left(\Omega^{\prime}\right) \geq \mu_{\mathcal{C}}(\Omega)$, et dont le bord $\partial \Omega^{\prime}$ est contenu dans le bord de $\Omega$ et vérifie donc $\nu_{\mathcal{C}}\left(\partial \Omega^{\prime}\right) \leq \nu_{\mathcal{C}}(\partial \Omega)$. Ainsi, on a

$$
\frac{\nu_{\mathcal{C}}\left(\partial \Omega^{\prime}\right)}{\mu_{\mathcal{C}}\left(\Omega^{\prime}\right)} \leq \frac{\nu_{\mathcal{C}}(\partial \Omega)}{\mu_{\mathcal{C}}(\Omega)}
$$

Il suffit donc de faire la preuve pour les domaines simplement connexes.

Soit donc $\Omega$ un domaine simplement connexe dont le bord $\partial \Omega$ est une courbe rectifiable $c: S^{1} \rightarrow \mathcal{C}$.

Alors, puisque nous sommes en dimension 2, chaque partie du $\varepsilon$-remplissage peut être recouvert par une boule de rayon $\varepsilon$. Pour préciser cela, rappelons qu'un $\varepsilon$-remplissage est la donnée d'une triangulation $P$ du disque $D^{2}$ et d'une application (non nécessairement continue) $\Phi$ de $D^{2}$ dans $\Omega$, qui applique le bord $S^{1}$ de $D^{2}$ sur le bord $\partial \Omega$ de $\Omega$, et telle que l'image de chaque face fermée de la triangulation $P$ a un diamètre au plus $\varepsilon$. Comme ici $\Omega$ est un disque topologique de $\mathbb{R}^{2}$, l'image des sommets de la triangulation doit être $\varepsilon$-dense dans $\Omega$. En effet, on peut étendre affinement la restriction de $\Phi$ aux sommets de la triangulation en une application continue $\tilde{\Phi}$ du disque dans $\Omega$ dont la restriction au bord est égal à l'application $c$. Supposons que l'image des sommets de la triangulation ne soit pas $\varepsilon$-dense. Alors, il existe un point $Q$ de $\Omega$ qui n'est pas dans l'image de $\tilde{\Phi}$. Cela provient du fait que l'image par $\tilde{\Phi}$ d'une face est

TOME $134-2006-\mathrm{N}^{\mathrm{O}} 3$ 
le triangle déterminé par l'image des sommets de la face considérée, qui est, par construction, de diamètre au plus $\varepsilon$. L'application $\tilde{\Phi}$ ne serait alors pas surjective, ce qui permettrait de rétracter le disque continûment sur son bord.

Cela permet de recouvrir $\Omega$ par un nombre de boules de rayon $\varepsilon$ égal au nombre de faces de $P$. On en déduit donc que

$$
\mu_{\mathcal{C}}(\Omega) \leq \operatorname{Aire}_{\varepsilon}(\partial \Omega) C_{2}(\varepsilon, n) \leq A^{\prime} \nu_{\mathcal{C}}(\partial \Omega)+B^{\prime}
$$

et ainsi

$$
\frac{\nu_{\mathcal{C}}(\partial \Omega)}{\mu_{\mathcal{C}}(\Omega)} \geq \frac{1}{A^{\prime}}-\frac{B^{\prime}}{\mu_{\mathcal{C}}(\Omega)} .
$$

Ceci nous donne une minoration de $I_{\infty}(\Omega)$ pour les domaines $\Omega$ de volume $\mu_{\mathcal{C}}(\Omega) \geq 2 A^{\prime} B^{\prime}$. Pour les domaines tels $\mu_{\mathcal{C}}(\Omega) \leq 2 A^{\prime} B^{\prime}$, on remarque que leur longueur est au moins égale à deux fois leur diamètre et que le volume d'une boule de rayon le diamètre, contenant le domaine est plus grand que celui du domaine. Le théorème 2.2 permet de conclure que $I_{\infty}(\mathcal{C})>0$.

Il reste donc à en déduire des informations pour le bas du spectre $\lambda_{1}(\mathcal{C})$. Classiquement, dans le cas des variétés riemanniennes, cela se fait via l'inégalité de Cheeger qui relie le bas du spectre et la constante de Cheeger. Cependant, dans le contexte des espaces métriques, il n'est pas clair qu'une telle relation existe en toute généralité. Aussi allons nous nous ramener dans un cadre où on sait qu'une telle inégalité existe : celui des géométries de Finsler. On sait que la distance de Hilbert dérive d'une métrique de Finsler, mais cette dernière a la régularité du bord du convexe considéré $\mathcal{C}$. Le but est de démontrer le théorème 2.7 ci-dessous, en se ramenant à une inégalité de Cheeger classique dans un cadre finslérien qui sera énoncée au théorème 2.11 et démontrée dans la section 4 .

ThÉORÈme 2.7. - Soit $\left(\mathcal{C}, d_{\mathcal{C}}\right)$ un convexe du plan muni de sa métrique de Hilbert. Alors il existe une constante $C>0$, ne dépendant pas de $\mathcal{C}$, telle que

$$
\lambda_{1}(\mathcal{C}) \geq C I_{\infty}^{2}(\mathcal{C})
$$

On en déduit le corollaire immédiat suivant :

Corollaire 2.8. - Soit $\left(\mathcal{C}, d_{\mathcal{C}}\right)$ un convexe du plan muni de sa métrique de Hilbert. Si $\lambda_{1}(\mathcal{C})=0$, alors $I_{\infty}(\mathcal{C})=0$.

Cela implique également le corollaire suivant, déjà démontré par A. Karlsson et G.A. Noskov [11] et par Y. Benoist [1] (voir lemme 2.12 et corollaire 2.13).

Corollaire 2.9. - Soit $\left(\mathcal{C}, d_{\mathcal{C}}\right)$ un convexe du plan muni de sa métrique de Hilbert qui est $\delta$-hyperbolique. Alors son bord est $C^{1}$ et strictement convexe. 
Démonstration. - Dans le cas contraire, on utilise à nouveau le résultat de Benzécri (voir [1], cor. 2.9, dû à Benzécri [2]), le convexe $\mathcal{C}$ admet un triangle dans son adhérence, sous l'action de $G_{2}$. On a vu que cela impliquait le fait que $\lambda_{1}(\mathcal{C})=0$ (voir démonstration de la proposition 1.3).

Le corollaire précédent implique que la constante de Cheeger $I_{\infty}(C)$ est également nulle. Ceci est en contradiction avec la proposition 2.5.

Remarquons enfin que l'on obtient également le théorème suivant.

Corollaire 2.10. - Soit $\left(\mathcal{C}, d_{\mathcal{C}}\right)$ un convexe du plan muni de sa métrique de Hilbert. Alors sa constante de Cheeger $I_{\infty}(C)$ est non nulle si et seulement s'il est Gromov-hyperbolique.

Ce corollaire est à comparer au résultat de Cao [5] dans le cas riemannien.

L'idée de la preuve du théorème 2.7 est de remplacer l'étude de la relation entre le bas du spectre de $\mathcal{C}, \lambda_{1}(\mathcal{C})$, et la constante de Cheeger de $\mathcal{C}, I_{\infty}(\mathcal{C})$, par l'étude des mêmes objets pour des domaines ouverts $\Omega$ tels que $\bar{\Omega} \subset \mathcal{C}$. Cela permettra de se ramener à une inégalité classique du type Cheeger dans le cas finslérien, puis de conclure par passage à la limite.

Le premier point est donc de définir la constante de Cheeger d'un domaine ouvert $\Omega$ de $\mathcal{C}$ avec $\bar{\Omega} \subset \mathcal{C}:$ on pose

$$
I_{\infty}^{\mathcal{C}}(\Omega)=\inf _{U \subset \Omega} \frac{\nu_{\mathcal{C}}(\partial U)}{\mu_{\mathcal{C}}(U)}
$$

où $U$ est un domaine dont l'adhérence (prise dans $\mathbb{R}^{2}$ ) vérifie $\bar{U} \subset \Omega$ et dont le bord $\partial U$ est une courbe rectifiable de longueur $\nu_{\mathcal{C}}(\partial U)$.

Le point décisif de la preuve à venir sera l'inégalité de Cheeger qui est valable pour des métriques de Finsler suffisamment régulières. On utilise le résultat suivant, qui est une application dans notre cadre du théorème plus général démontré dans la section 4.

ThÉoRème 2.11. - Soit $\left(\mathcal{C}, d_{\mathcal{C}}\right)$ un convexe du plan muni de sa métrique de Hilbert. Si le bord de $\mathcal{C}$ est $C^{1}$ et strictement convexe, il existe une constante $C>0$ telle que pour tout ouvert $\Omega$ on a

$$
\lambda_{1}^{\mathcal{C}}(\Omega) \geq C\left(I_{\infty}^{\mathcal{C}}\right)^{2}(\Omega) .
$$

En particulier cela implique que

$$
\lambda_{1}(\mathcal{C}) \geq C I_{\infty}^{2}(\mathcal{C})
$$

Remarquons que la constante $C$ est universelle; elle ne dépend en fait que de la dimension, comme on le verra au théorème 4.1.

TOME $134-2006-\mathrm{N}^{\mathrm{O}} 3$ 
Démonstration du théorème 2.7 à l'aide du théorème 2.11.

La seule difficulté, pour passer du théorème 2.11 au théorème 2.7 est que dans ce dernier, le bord du convexe $\mathcal{C}$ n'est pas nécessairement de classe $C^{1}$ : il faut donc se ramener à cette situation.

Soit $\Omega$ un domaine du plan tel que $\bar{\Omega} \subset \mathcal{C}$. Comme dans les deux lemmes 1.4 et 1.5, il existe des homothétiques $(1+\rho) \mathcal{C}$ et $(1-\rho) \mathcal{C}$ de $\mathcal{C}$ tels que $\bar{\Omega} \subset(1-2 \rho) \mathcal{C}$, et tels que pour tout convexe $\Gamma$ vérifiant

$$
(1-\rho) \mathcal{C} \subset \Gamma \subset(1+\rho) \mathcal{C}
$$

on ait

$$
\lim _{\rho \rightarrow 0} \lambda_{1}^{\Gamma}(\Omega)=\lambda_{1}^{\mathcal{C}}(\Omega) .
$$

Mais la preuve des lemmes 1.4 et 1.5 montre que la même chose est vraie pour la constante de Cheeger, c'est-à-dire que pour tout domaine $\Gamma$ vérifiant

$$
(1-\rho) \mathcal{C} \subset \Gamma \subset(1+\rho) \mathcal{C}
$$

on a

$$
\lim _{\rho \rightarrow 0} I_{\infty}^{\Gamma}(\Omega)=I_{\infty}^{\mathcal{C}}(\Omega) .
$$

On fait alors tendre $\rho$ vers 0 tout en choisissant une famille $\Gamma_{\rho}$ d'ensembles convexes de classe $C^{1}$, strictement convexes, tels que

$$
(1-\rho) \mathcal{C} \subset \Gamma_{\rho} \subset(1+\rho) \mathcal{C} .
$$

Pour montrer qu'une telle famille existe, on remplace dans un premier temps le convexe $\mathcal{C}$ par un polygone convexe $\mathcal{C}^{\prime}$ approximant $\mathcal{C}$ de sorte à vérifier

$$
\left(1-\frac{1}{2} \rho\right) \mathcal{C} \subset \mathcal{C}^{\prime} \subset\left(1+\frac{1}{2} \rho\right) \mathcal{C},
$$

puis on remplace chaque segment de $\mathcal{C}^{\prime}$ par un arc strictement convexe, de façon à avoir un recollement de classe $C^{1}$ en chaque sommet. Cela peut se faire en approximant $\mathcal{C}^{\prime}$ d'aussi prêt que l'on veut, à condition de faire l'approximation avec des fonctions polynomiales de degré élevé.

Dès lors, on peut appliquer l'inégalité (12)

$$
\lambda_{1}^{\Gamma_{\rho}}(\Omega) \geq C\left(I_{\infty}^{\Gamma_{\rho}}\right)^{2}(\Omega)
$$

et, en passant à la limite, on obtient

$$
\lambda_{1}^{\mathcal{C}}(\Omega) \geq C\left(I_{\infty}^{\mathcal{C}}\right)^{2}(\Omega) .
$$

Pour conclure, il suffit donc de passer à la limite sur les $\Omega$; si l'inégalité ci-dessus n'était pas vraie pour $\mathcal{C}$ à la place de $\Omega$, il existerait un domaine $\Omega$ pour lequel elle ne serait pas valide non plus, puisque la constante de Cheeger et le bas du spectre de $\mathcal{C}$ sont approximables d'aussi prêt que l'on veut par la constante de Cheeger et le bas du spectre des domaines d'adhérence compacte dans $\mathcal{C}$, voir lemme 1.4 . 
REMARQUE. - Dans notre preuve nous approchons par une famille de convexes dont le bord est de classe $C^{1}$ et strictement convexe, ce qui nécessite l'adaptation à ce cadre de l'inégalité (12). Un résultat de L. Hörmander [10], précisément le lemme 2.3.2, permet d'obtenir une famille d'ensemble de classe $C^{2}$, dont le bord admet un hessien strictement positif en tout point. Dans ce cadre l'inégalité (12) est classique, voir par exemple Z. Shen [12].

Le théorème 2.1 est à présent un corollaire du théorème 2.7 qui contrôle le bas du spectre $\lambda_{1}(\mathcal{C})$ par la constante de Cheeger $I_{\infty}(\mathcal{C})$ et de la proposition 2.5 qui contrôle la constante de Cheeger en fonction du $\delta$ de la $\delta$-hyperbolicité.

\section{Formule de la co-aire}

Afin de démontrer une version très générale du théorème 2.11 , on a besoin d'une formule de la co-aire. Ce qui suit apporte les outils et notions nécessaires à sa démonstration et son expression : dans une variété de Finsler $(M, F)$ munie d'une mesure $\mu$, il s'agit d'une part de définir une mesure naturellement associée à $\mu$ sur les hypersurfaces de $M$ (sous-section 3.1), puis de préciser la notion de gradient (sous-section 3.2). A la section 4, on appliquera cela pour la mesure de Hausdorff associée à la métrique de Finsler de la variété.

Cette section combine des idées contenues dans les livres de A. Thompson [15], chap. 3 et 5 , et Z. Shen [12], chap. 3 et 4.

3.1. Mesure sur les hypersurfaces. - Soit $(V, F)$ un espace vectoriel de dimension finie muni d'une norme $F$, dont la sphère unité $S_{F}(0,1)$ centrée en 0 est $C^{1}$ et strictement convexe.

DÉfinition 3.1. - On dira que $x$ est normal à $y$, ce que l'on notera $x \dashv y$, si et seulement si, pour tout $\alpha \in \mathbb{R}$ on a

$$
F(x+\alpha y) \geq F(x)
$$

Remarque. - Cette relation n'est généralement pas symétrique.

Soit $y$ un vecteur non nul. On lui associe l'hyperplan

$$
W_{y}:=\{w \in V \mid y \dashv w\} .
$$

Considérons une base $\left\{b_{i}\right\}_{i=2, \ldots, n}$ de $W_{y}$ et $b_{1}=y$. Ainsi $\left\{b_{i}\right\}_{i=1}^{n}$ est une base de $V$. Définissons alors

$$
\begin{aligned}
B^{n}\left(b_{1}, \ldots, b_{n}\right) & :=\left\{\left(y^{i}\right)_{i=1}^{n} \in \mathbb{R}^{n} ; F\left(\sum_{i=1}^{n} y^{i} b_{i}\right)<1\right\} \text { et } \\
B_{y}^{n-1}\left(b_{2}, \ldots, b_{n}\right) & :=\left\{\left(y^{j}\right)_{j=2}^{n} \in \mathbb{R}^{n-1} ; F\left(\sum_{j=2}^{n} y^{j} b_{j}\right)<1\right\} .
\end{aligned}
$$

Les deux domaines $B^{n}$ et $B_{y}^{n-1}$ dépendent du choix de la base $\left\{b_{j}\right\}_{j=2}^{n}$.

TOME $134-2006-\mathrm{N}^{\mathrm{O}} 3$ 
On définit la fonction

$$
\zeta(y):=\frac{\operatorname{vol}_{e}\left(\mathbb{B}^{n}\right)}{\operatorname{vol}_{e}\left(\mathbb{B}^{n-1}\right)} \cdot \frac{\operatorname{vol}_{e}\left(B_{y}^{n-1}\left(b_{2}, \ldots, b_{n}\right)\right)}{F(y) \operatorname{vol}_{e}\left(B^{n}\left(b_{1}, \ldots, b_{n}\right)\right)} .
$$

Remarquons, que si $F$ est euclidienne, alors $\zeta(y)=1$.

De plus, puisque pour tout réel positif $\lambda>0, F(\lambda y)=\lambda F(y)$,

$$
\begin{aligned}
\operatorname{vol}_{e}\left(B^{n}\left(\lambda y, b_{2}, \ldots, b_{n}\right)\right) & =\operatorname{vol}_{e}\left(B^{n}\left(y, b_{2} / \lambda, \ldots, b_{n} / \lambda\right)\right) \lambda^{n} \text { et } \\
\operatorname{vol}_{e}\left(B^{n-1}\left(b_{2}, \ldots, b_{n}\right)\right) & =\operatorname{vol}_{e}\left(B^{n-1}\left(b_{2} / \lambda, \ldots, b_{n} / \lambda\right)\right) \lambda^{n-1}
\end{aligned}
$$

on a dans tous les cas la propriété suivante :

$$
\zeta(\lambda y)=\zeta(y), \quad \lambda>0, y \neq 0 .
$$

Lemme 3.2. - Soit $(V, F)$ un espace vectoriel normé et

$$
c_{n}:=\operatorname{vol}_{e}\left(\mathbb{B}^{n}\right) / \operatorname{vol}_{e}\left(\mathbb{B}^{n-1}\right) .
$$

Alors pour y non nul on a

$$
2^{-n} c_{n} \leq \zeta(y) \leq 2^{n} c_{n} .
$$

Démonstration. - Soit $y=\sum_{i=1}^{n} y^{i} b_{i}$ un vecteur normé de $(V, F)$ et considérons la boule $B_{y}^{n-1}$. Alors pour tout $t \in[0,1]$ et $\left(v^{j}\right)_{j=2}^{n} \in B_{y}^{n-1}$, par l'inégalité triangulaire, on a

$$
F\left(t y+\sum_{j=2}^{n} v^{j} b_{j}\right) \leq t F(y)+F\left(\sum_{j=2}^{n} v^{j} b_{j}\right) \leq 2
$$

donc $[0,1] \times B_{y}^{n-1} \subset 2 B^{n}$. De ceci on déduit que

$$
\operatorname{vol}_{e n-1}\left(B_{y}^{n-1}\right) \leq 2^{n} \operatorname{vol}_{e}\left(B^{n}\right) .
$$

Soit à présent $\left(t,\left(v^{j}\right)_{j=2}^{n}\right) \in B^{n}$. Autrement dit $F\left(t y+\sum_{j=2}^{n} v^{j} b_{j}\right) \leq 1$ et $\sum_{j=2}^{n} v^{j} b_{j} \in W_{y}$, soit $y \dashv \sum_{j=2}^{n} v^{j} b_{j}$. La normalité donne $(t \neq 0)$

$$
|t|=F(t y)=F(|t| y) \leq F\left(|t| y+(|t| / t) \sum_{j=2}^{n} v^{j} b_{j}\right) \leq 1
$$

et, grâce à l'inégalité triangulaire, on obtient

$$
F\left(\sum_{j=2}^{n} v^{j} b_{j}\right) \leq F\left(t y+\sum_{j=2}^{n} v^{j} b_{j}\right)+F(-t y) \leq 2
$$

ceci implique que $B^{n} \subset[-1,1] \times 2\left[B_{y}^{n-1}\right]$, et donc

$$
\operatorname{vol}_{e}\left(B^{n}\right) \leq 2^{n} \operatorname{vol}_{e n-1}\left(B_{y}^{n-1}\right) .
$$

BULLETIN DE LA SOCIÉtÉ MATHÉMATIQUE DE FRANCE 
On peut maintenant définir une mesure adéquate sur une hypersurface $N$ d'une variété de Finsler $(M, F)$. Sur $M$, on peut considérer la mesure de Hausdorff associée à $F$, que l'on note $\mu_{F}$. Pour définir une mesure sur $N$, on considère la restriction de $F$ à $N$ qui muni $N$ d'une métrique de Finsler dont la mesure de Hausdorff associée est notée $\bar{\nu}_{F}$. Cette dernière est en apparence le candidat naturel à être la mesure associée à $\mu_{F}$ sur $N$. C'est d'ailleurs la mesure que l'on a considérée à la sous-section 2.2 pour définir la constante de Cheeger $I_{\infty}(\mathcal{C})$ en dimension 2. Cependant, la suite de ce paragraphe, notamment la preuve de la formule de la co-aire, montre qu'un choix un peu différent peut être plus adéquat. On introduit un champ de vecteurs normaux unités $n$ sur $N$. Cela signifie que pour tout point $p \in N$, l'hyperplan $T_{p} N$ tangent à $N$ au point $p$ est normal à $n(p)$ au sens défini ci-dessus, c'est-à-dire

$$
T_{p} N:=\left\{w \in T_{p} M \mid n(p) \dashv w\right\} .
$$

On choisit alors d'associer à $\mu_{F}$ la mesure

$$
\nu_{F}=\zeta(n) \bar{\nu}_{F} \text {. }
$$

Ainsi, en général, la mesure $\nu_{F}$ ne coïncide pas avec $\bar{\nu}_{F}$, mais c'est le cas lorsque la métrique est riemannienne. Remarquons que, par la propriété 3.2, ces deux mesures $\bar{\nu}_{F}$ et $\nu_{F}$ sont dans un rapport borné, uniformément contrôlé, ce qui sera crucial à la section 4, puisque l'on utilisera $\bar{\nu}_{F}$ pour définir la constante de Cheeger et $\nu_{F}$ pour la formule de la co-aire.

Cette définition, pour le cas particulier de la mesure de Hausdorff, se généralise comme suit :

DÉfinition 3.3. - Soit $N$ une hypersurface dans un espace de Finsler $(M, F)$. Si $\mu$ est une mesure sur $M$ absolument continue par rapport à la mesure de Hausdorff $\mu_{F}$, i.e. on peut écrire $\mu(p)=\phi(p) \mu_{F}(p)$ en tout point $p$ de $M$, on associe à $\mu$ la mesure $\nu$ sur l'hypersurface $N$ définie, pour tout $p \in N$, par

$$
\nu(p)=\phi(p) \nu_{F}(p) .
$$

Remarquons que l'on doit introduire la pondération par la fonction $\zeta$ lorsqu'on cherche à obtenir la propriété naturelle suivante.

Lemme 3.4. - Si $V_{F}$ est le volume de Hausdorff induit par $F$ sur $M$, alors

$$
A_{F}(S(x, \rho))=\lim _{\varepsilon \rightarrow 0} \frac{V_{F}(B(x, \rho+\varepsilon))-V_{F}(B(x, \rho))}{\varepsilon} .
$$

3.2. Gradient d'une fonction par rapport à une norme strictement convexe. - On voudrait associer à la différentielle $\mathrm{d} f_{x}$ d'une fonction en $x \in M^{n}$ un vecteur $X$ dans l'espace tangent $T_{x} M$ tel que

$$
F^{*}\left(\mathrm{~d} f_{x}\right)=F(X) .
$$

TOME $134-2006-\mathrm{N}^{\mathrm{O}} 3$ 
Pour ceci, on prend le point $X \in S_{F}\left(0, F^{*}\left(d f_{x}\right)\right)$, sur la sphère de centre 0 et de rayon $F^{*}\left(\mathrm{~d} f_{x}\right)$, tel que

$$
\text { ker } \mathrm{d} f_{x}=\{v \mid X \dashv v\} \quad \text { et } \quad \mathrm{d} f_{x}(X)>0
$$

(l'hyperplan $X+\operatorname{ker} \mathrm{d} f_{x}$ est l'hyperplan d'appui de $S\left(0, F^{*}\left(\mathrm{~d} f_{x}\right)\right)$ en X).

On remarquera qu'en raison de la stricte convexité

$$
\mathrm{d} f_{x}(X)=F^{*}\left(\mathrm{~d} f_{x}\right) F(X)=F^{*}\left(\mathrm{~d} f_{x}\right)^{2} .
$$

On dira que $X$ est le gradient de $f$ en $x$ et on le notera $\nabla f(x)$.

Remarquons que, pour une fonction $C^{1}$, la fonction $x \mapsto \nabla f(x)$ est continue et que, pour une fonction au moins $C^{2}$, elle est $C^{1}$ puisque la sphère $S(0,1)$ est $C^{1}$.

Lemme 3.5 (lemme de Gauss finslérien). - Soit $f$ une fonction $C^{2}$ sur un ouvert $U$ tel que $\mathrm{d} f \neq 0$. Soit $N_{t}=f^{-1}(t)$. Alors le vecteur $n=\nabla f_{N_{t}} / F(\nabla f)$ est normal à $N_{t}$. Autrement dit, le gradient est normal aux lignes de niveaux.

Démonstration. - Puisque $f$ est constante sur la ligne de niveau $N_{t}$, on a

$$
\mathrm{d} f(w)=0, \quad \forall w \in T N_{t},
$$

et donc $\nabla f \dashv w$ pour tout $w \in T N_{t}$ par définition de $\nabla f$.

3.3. Démonstration de la formule de la co-aire. - On démontre le théorème suivant.

THÉORÈme 3.6. - Soit $\left(M^{n}, F, \mu\right)$ une variété finslérienne mesurée, telle qu'en tout point $x \in M$ la boule unité de la norme $F$ est $C^{1}$ et strictement convexe. Soit $f$ une fonction $C^{1}$ par morceaux sur $M$ tel que pour tout $t$, l'ensemble $f^{-1}(t)$ est compact. Alors pour toute fonction continue $\varphi$ sur $M$ on a la formule de la co-aire suivante

$$
\int_{M} \varphi F^{*}(\mathrm{~d} f) \mathrm{d} \mu=\int_{-\infty}^{+\infty} \int_{f^{-1}(t)} \varphi \mathrm{d} \nu \mathrm{d} t,
$$

où $\nu$ désigne la mesure associée à $\mu$ sur l'hypersurface $f^{-1}(t)$ selon la définition 3.3.

On va faire une démonstration partielle de ce résultat classique, en géométrie riemannienne, dans le cas où les lignes de niveau $\left\{f^{-1}(t)\right\}$ sont des sous-variétés, cela pour montrer comment la mesure $\nu$ que l'on a introduit sur les hypersurfaces entre naturellement en jeu. Par le théorème de Sard, on sait qu'il existe un ensemble de mesure nulle de points $t$ pour lesquels $\left\{f^{-1}(t)\right\}$ n'est pas une sous-variété. On s'inspire de la démonstration de [12]. 
Démonstration. - On va faire la preuve dans une carte $U$. Pour simplifier, on supposera que d $f \neq 0$ sur $U$ et que $f$ est $C^{2}$. Fixons un nombre $t_{0}$ tel que $f^{-1}\left(t_{0}\right) \cap U \neq 0$ et soit $X$ le champ de vecteurs défini sur $U$ par

$$
X=\frac{\nabla f}{F^{*}(\mathrm{~d} f)^{2}} .
$$

On commence par relever le flot. Pour un point $x \in f^{-1}\left(t_{0}\right) \cap U$, soit $c(t)$ la courbe intégrale de $X$ telle que $c\left(t_{0}\right)=x$ (on peut parler de « la » courbe car $X$ est $C^{1}$ sur $U$ ). Alors

$$
\frac{\mathrm{d}}{\mathrm{d} t}[f \circ c(t)]=\mathrm{d} f(X)=\frac{\mathrm{d} f(\nabla f)}{F^{*}(\mathrm{~d} f)^{2}}=1
$$

par construction de $\nabla f$ et par conséquent

$$
f \circ c(t)=t \text {. }
$$

Les courbes intégrales de $X$ donnent naissance à un système de coordonnées $\left.\psi=\left(x^{1}, \ldots, x^{n}\right): U \rightarrow\right]-\varepsilon, \varepsilon\left[\times \mathbb{B}^{n-1}\right.$ tel que

$$
f \circ \psi^{-1}\left(x^{1},\left(x^{i}\right)_{i=2}^{n}\right)=x^{1} .
$$

Définissons l'ensemble $N_{t}$ par

$$
f^{-1}(t) \cap U=\psi^{-1}\left(t \times \mathbb{B}^{n-1}\right) .
$$

Suivant le lemme de Gauss finslérien, le champ de vecteurs

$$
n=\frac{\nabla f}{F^{*}(\mathrm{~d} f)}
$$

est normal à $N_{t}$. Considérons donc une base locale $\left\{b_{i}\right\}_{i=1, \ldots, n}$ de $T M$ avec $b_{1}=n$ et $b_{i}=\partial / \partial x^{i}$ pour $i=2, \ldots, n$. Soit $\left(\theta^{i}\right)_{i=1, \ldots, n}$ la base duale sur $T^{*} M$. On a alors

$$
\theta^{1}=\lambda \mathrm{d} x^{1}=\lambda \mathrm{d} f, \quad \theta^{i}=\mathrm{d} x^{i},(i=2, \ldots, n) .
$$

Remarquons également que

$$
1=\theta^{1}(n)=\lambda \mathrm{d} f\left(\frac{\nabla f}{F^{*}(\mathrm{~d} f)}\right)=\lambda F^{*}(\mathrm{~d} f),
$$

ce qui détermine $\lambda$, i.e.

$$
\theta^{1}=\frac{1}{F^{*}(\mathrm{~d} f)} \mathrm{d} x^{1} .
$$

À présent, si $\mathrm{d} \mu=\sigma(x) \theta^{1} \wedge \cdots \wedge \theta^{n}$, alors par construction

$$
\mathrm{d} \nu=\sigma(x) \hat{\theta}^{2} \wedge \cdots \wedge \hat{\theta}^{n}
$$

TOME $134-2006-\mathrm{N}^{\mathrm{O}} 3$ 
où les $\hat{\theta}^{i}$ sont les tirés en arrière des $\theta^{i}$ sur $N_{t}$. Autrement dit

$$
\begin{aligned}
\mathrm{d} \mu & =\frac{\sigma(x)}{F^{*}(\mathrm{~d} f)} \mathrm{d} x^{1} \wedge \cdots \wedge \mathrm{d} x^{n}, \\
\mathrm{~d} \nu & =\sigma(x) \mathrm{d} x^{2} \wedge \cdots \wedge \mathrm{d} x^{n} .
\end{aligned}
$$

REMARQUE. - Si d $\mu$ est la mesure de finsler $n$-dimensionnelle, alors on voit ici apparaître naturellement la fonction $\zeta$ puisque $\mathrm{d} \nu$ est exactement $\zeta$ fois la mesure de finsler $n-1$ dimensionnelle.

Il reste à intégrer sur $U$ :

$$
\begin{aligned}
\int_{U} \varphi F^{*}(\mathrm{~d} f) \mathrm{d} \mu & =\int_{]-\varepsilon, \varepsilon\left[\times \mathbb{B}^{n-1}\right.} f \sigma(x) \mathrm{d} x^{1} \cdots \mathrm{d} x^{n} \\
& =\int_{-\varepsilon}^{\varepsilon}\left(\int_{\mathbb{B}^{n-1}} f \sigma(x) \mathrm{d} x^{2} \cdots \mathrm{d} x^{n}\right) \mathrm{d} x^{1}=\int_{-\varepsilon}^{\varepsilon}\left(\int_{N_{t} \cap U} f \mathrm{~d} \nu\right) \mathrm{d} t .
\end{aligned}
$$

\section{Inégalité de Cheeger finslérienne}

Dans cette section, on va démontrer une version $n$-dimensionelle du théorème 2.11 :

ThÉORÈme 4.1. - Soit $\left(\mathcal{C}, d_{\mathcal{C}}\right)$ un domaine convexe de $\mathbb{R}^{n}$ muni de sa métrique de Hilbert. Si le bord de $\mathcal{C}$ est $C^{1}$ et strictement convexe, il existe une constante $C=C(n)>0$ telle que pour tout ouvert $\Omega$ on a

$$
\lambda_{1}^{\mathcal{C}}(\Omega) \geq C\left(I_{\infty}^{\mathcal{C}}\right)^{2}(\Omega) .
$$

En particulier cela implique que

$$
\lambda_{1}(\mathcal{C}) \geq C I_{\infty}^{2}(\mathcal{C})
$$

Ce théorème sera une conséquence immédiate de l'inégalité de Cheeger en géométrie de Finsler. Si $(M, F)$ est une variété de Finsler de classe $C^{1}$ de dimension $n, \mu_{F}$ la mesure de Hausdorff associée à $F$, on désigne par $\lambda_{1}(M, F)$ le bas du spectre de $(M, F)$ défini par

$$
\lambda_{1}(M, F)=\inf _{f} \frac{\int_{M} F^{*}(\mathrm{~d} f)^{2} \mathrm{~d} \mu_{F}}{\int_{\Omega}|f|^{2} \mathrm{~d} \mu_{F}},
$$

où $f$ est une fonction lipschitzienne à support compact dans $M$. On désigne par $I_{\infty}(M, F)$ la constante de Cheeger de $(M, F)$ définie par

$$
I_{\infty}(M, F)=\inf _{U} \frac{\bar{\nu}_{F}(\partial U)}{\mu_{F}(U)}
$$

où $U$ décrit les ouverts de $M$ d'adhérence compacte et dont le bord $\partial U$ est une sous-variété de dimension $n-1$. On rappelle que $\bar{\nu}_{F}$ désigne la mesure 
de Hausdorff des hypersurfaces associée à la restriction de $F$. Alors, dans ces conditions

ThÉoRÈme 4.2 (Inégalité de Cheeger finslerienne). - Il existe une constante $C=C(n)>0$ telle que

$$
\lambda_{1}(M, F) \geq C I_{\infty}^{2}(M, F) .
$$

De manière générale, soient $\left(M^{n}, F, \mu\right)$ une variété finslérienne mesurée et $\nu$ la mesure induite sur les hypersurfaces au sens de la définition 3.3.

Considérons un domaine $\Omega$ de $M$ d'adhérence compacte. On définit la constante de Sobolev $S_{\infty}(\Omega)$ de $\Omega$ par

$$
S_{\infty}(\Omega)=\inf _{f} \frac{\int_{\Omega} F^{*}(\mathrm{~d} f) \mathrm{d} \mu}{\int_{\Omega}|f| \mathrm{d} \mu}
$$

pour $f$ une fonction lipschitzienne à support compact dans $\Omega$. On définit de manière similaire $S_{\infty}(M)$ par

$$
S_{\infty}(M)=\inf _{f} \frac{\int_{M} F^{*}(\mathrm{~d} f) \mathrm{d} \mu}{\int_{M}|f| \mathrm{d} \mu}
$$

pour $f$ une fonction lipschitzienne à support compact dans $M$.

On peut associer aux mesures $\mu$ et $\nu$ une constante de Cheeger sur $\Omega$ et sur $M$ définie par

$$
I_{\infty}^{M, \mu}(\Omega)=\inf _{U} \frac{\nu(\partial U)}{\mu(U)} \quad\left(\operatorname{resp} . I_{\infty}^{\mu}(M)=\inf _{U} \frac{\nu(\partial U)}{\mu(U)}\right)
$$

où $U$ est un domaine tel que $\bar{U} \subset \Omega$ (resp. $\bar{U} \subset M$ ) et dont le bord $\partial U$ est une sous-variété de dimension $n-1$.

Comme on l'a dit, lorsque $\mu=\mu_{F}$ est la mesure de Hausdorff associée à $F$, on a $I_{\infty}^{\mu_{F}}(M) \neq I_{\infty}(M, F)$ puisque $\nu_{F} \neq \bar{\nu}_{F}$.

L'inégalité de Cheeger est une conséquence du

ThÉORÈme 4.3. - On a les égalités $I_{\infty}^{M, \mu}(\Omega)=S_{\infty}(\Omega)$ et $I_{\infty}^{\mu}(M)=S_{\infty}(M)$.

En effet, en considérant $f=h^{2}$ et en appliquant l'inégalité de CauchySchwarz, on a

$$
\left(I_{\infty}^{\mu_{F}}\right)^{2}(M) \leq 4 \inf _{h} \frac{\int_{M} F^{*}(\mathrm{~d} h)^{2} \mathrm{~d} \mu_{F}}{\int_{M}|h|^{2} \mathrm{~d} \mu_{F}}=\lambda_{1}(M, F) .
$$

Il reste alors à comparer $I_{\infty}^{\mu_{F}}(M)$ à la constante de Cheeger $I_{\infty}(M, F)$ que l'on considère dans la première partie. Cela vient de l'inégalité (3.2) qui nous permet d'obtenir l'inégalité

$$
I_{\infty}(M) c_{1}(n) \leq I_{\infty}^{\mu_{F}}(M) \leq I_{\infty}(M) c_{2}(n) .
$$

TOME $134-2006-\mathrm{N}^{\mathrm{O}} 3$ 
Démonstration du théorème 4.3. — On suit la démonstration du théorème 6.2 dans [6], p. 266-269. On va démontrer que $I_{\infty}^{\mu}(M)=S_{\infty}(M)$ à l'aide de deux inégalités.

Première étape : on montre que $S_{\infty}(M) \leq I_{\infty}^{\mu}(M)$. - Soit donc $U$ un domaine ouvert de $M$ d'adhérence compacte et bord lisse. Pour tout $\varepsilon$ suffisamment petit on considère la fonction

$$
f_{\varepsilon}(x)= \begin{cases}1 & \text { si } x \in U, \\ \varepsilon^{-1} d(x, \partial U) & \text { si } x \in M \backslash U, d(x, \partial U)<\varepsilon, \\ 0 & \text { si } x \in M \backslash U, d(x, \partial U) \geq \varepsilon,\end{cases}
$$

en sorte que $f_{\varepsilon}$ est lipschitzienne. Donc

$$
S_{\infty}(M) \leq \frac{\int_{M} F^{*}\left(\mathrm{~d} f_{\varepsilon}\right) \mathrm{d} \mu}{\int_{M}\left|f_{\varepsilon}\right| \mathrm{d} \mu} .
$$

On obtient par construction

$$
\lim _{\varepsilon \rightarrow 0} \int_{M}\left|f_{\varepsilon}\right| \mathrm{d} \mu=\mu(U) .
$$

De plus, on a

$$
F^{*}\left(\mathrm{~d} f_{\varepsilon}\right)= \begin{cases}1 / \varepsilon & \text { si } x \in M \backslash \bar{U}, d(x, \partial U) \leq \varepsilon, \\ 0 & \text { sinon, }\end{cases}
$$

ce qui implique (voir section 3.1)

$$
\lim _{\varepsilon \rightarrow 0} \int_{M} F^{*}\left(\mathrm{~d} f_{\varepsilon}\right) \mathrm{d} \mu=\lim _{\varepsilon \rightarrow 0} \frac{\mu(\{x \notin U d(x, \partial U) \leq \varepsilon\})}{\varepsilon}=\nu(\partial U),
$$

et par conséquent, on en déduit que pour tout $U$

$$
S_{\nu}(M) \leq \frac{\nu(\partial U)}{\mu(U)}
$$

ce qui permet de conclure cette première étape en prenant l'infimum sur les $U$.

Seconde étape : on montre que $I_{\infty}^{\mu}(M) \leq S_{\infty}(M)$. — Soit $f$ une fonction lisse à support compact dans $M$. On pose

$$
U(t)=\{x ;|f|(x)>t\}, \quad \mu(t)=\mu(U(t)), \quad \nu(t)=\nu(\partial U(t)) .
$$

pour les $t$ atteints par $f$. Alors la formule de la co-aire (13) implique

$$
\int_{M} F^{*}(\mathrm{~d} f) \mathrm{d} \mu=\int_{0}^{+\infty} \nu(t) \mathrm{d} t \geq I_{\infty}^{\mu}(M) \int_{0}^{+\infty} \mu(t) \mathrm{d} t
$$

(où l'on a utilisé le fait que $F^{*}(\mathrm{~d} f)=F^{*}(\mathrm{~d}|f|)$ presque partout sur $M$ ). 
On remarque également que l'on a l'égalité

$$
\int_{M}|f| \mathrm{d} \mu=\int_{M} \int_{0}^{|f|} \mathrm{d} t \mathrm{~d} \mu=\int_{0}^{+\infty} \int_{U(t)} \mathrm{d} \mu \mathrm{d} t=\int_{0}^{+\infty} \mu(t) \mathrm{d} t
$$

qui permet d'arriver à l'inégalité

$$
I_{\infty}^{\mu}(M) \leq \frac{\int_{M} F^{*}(\mathrm{~d} f) \mathrm{d} \mu}{\int_{M}|f| \mathrm{d} \mu}
$$

et un passage à l'infimum sur toute les fonctions lisses à support compact $f$ permet de conclure.

\section{BIBLIOGRAPHIE}

[1] Benoist (Y.) - Convexes hyperboliques et fonctions quasisymétriques, Publ. Math. Inst. Hautes Études Sci., t. 97 (2003), pp. 181-237.

[2] BENZÉCRI (J.P.) - Sur les variétés localement affines et localement projectives, Bull. Soc. Math. France, t. 88 (1960), pp. 229-232.

[3] Bridson (M.R.) \& Haefliger (A.) - Metric spaces of non-positive curvature, Comprehensive Studies in Mathematics, vol. 319, Springer, 1999.

[4] Burago (D.), Burago (Y.) \& Ivanov (S.) - A course in metric geometry, Graduate Studies in Mathematics, vol. 33, Amer. Math. Soc., Providence, RI, 2001.

[5] CAO (J.) - Cheeger isoperimetric constants of Gromov-hyperbolic spaces with quasi-poles, Commun. Contemp. Math., t. 2 (2000), pp. 511-533.

[6] Chavel (I.) - Riemannian geometry : a modern introduction, Cambridge Tracts in Mathematics, vol. 108, Cambridge University Press, Cambridge, 1993.

[7] Colbois (B.), Vernicos (C.) \& Verovic (P.) - L'aire des triangles idéaux en géométrie de Hilbert, Enseign. Math., t. 50 (2004), no. 3-4, pp. 203-237.

[8] Colbois (B.) \& Verovic (P.) - Hilbert geometry for strictly convex domains, Geom. Dedicata, t. 105 (2004), pp. 29-42.

[9] DE LA HARPE (P.) - On Hilbert's metric for simplices, in Geometric group theory, Vol. 1 (Sussex, 1991), Cambridge Univ. Press, Cambridge, 1993, pp. 97-119.

[10] Hörmander (L.) - Notions of convexity, Progress in Mathematics, vol. 127, Birkhäuser Boston Inc., Boston, MA, 1994.

[11] Karlsson (A.) \& Noskov (G. A.) - The Hilbert metric and Gromov hyperbolicity, Enseign. Math. (2), t. 48 (2002), pp. 73-89.

[12] Shen (Zhongmin) - Lectures on Finsler geometry, World Scientific, 2001.

TOME $134-2006-\mathrm{N}^{\mathrm{O}} 3$ 
[13] Socié-MÉthou (E.) - Caractérisation des ellipsoïdes par leurs groupes d'automorphismes, Ann. Sci. École Norm. Sup. (4), t. 35 (2002), pp. 537548.

[14] _ Behaviour of distance functions in Hilbert-Finsler geometry, Differential Geom. Appl., t. 20 (2004), pp. 1-10.

[15] Thompson (A.C.) - Minkowski geometry, Encyclopedia of Mathematics and its applications, vol. 63, Cambridge University Press, 1996.

[16] Vernicos (C.) - The macroscopic sound of tori, Pacific J. Math., t. 213 (2004), pp. 121-156. 